\title{
THE NONLINEAR CAUSTIC
}

\author{
NG KIN-CHUNG
}

\begin{abstract}
In this paper the boundary layer at the nonlinear caustic is discussed and a monlinear Airy equation is,obtained by adapting techniques from linear theory. It is shown that the incident beam undergoes a phase shift by an amount of $\pi / 2$ as it passes through the caustic and becomes the outgoing beam.
\end{abstract}

\section{Introduction}

In the theory of nonlinear optics, one is led to consider a nonlinear reduced wave equation

$$
\nabla^{2} u+k^{2} n^{2}\left(|u|^{2}\right) u=0
$$

where $n=n\left(|u|^{2}\right)$ is a function of the intensity of the field and $n$ is called index of refraction.

Equation (1.1) is quite complicated. For the problems of optical phenomena, we assume that the wave frequency $\omega$ is high so that $k$ is large. In order to study (1.1) we use techniques which are developed from linear theory.

In the study of the linear version of equation (1.1) which is

$$
\nabla^{2} u+k^{2} n^{2} u=0
$$

explicit formulas for the solution of boundary value problem for (1.2) can occassionally be obtained but such results are generally not useful without further simplification. Therefore asymptotic methods have come to play an important role in the solution of problems for (1.2) in the ease where $k$ is large. This is precisely the case that occurs when dealing with optical phenomena. Write $u=a e^{i k \phi}$ where $\phi$ is the phase term and $a$ is the amplitude term. Substituting $u$ into (1.2) we are led 'o consider two equations

$$
\begin{gathered}
{\left[(\nabla \phi)^{2}-n^{2}\right] a-\frac{1}{k^{2}} \nabla^{2} a=0} \\
2 \nabla \phi \cdot \nabla a+a \nabla^{2} \phi=0 .
\end{gathered}
$$

Receive January 7, 1991.

1980 AMS(MOS) Suject Classfication: 30A32, 30A34, 30C45 
In the geometrical optics approximation we let $k \rightarrow \infty$ in (1.3) and obtain

$$
\begin{gathered}
(\nabla \phi)^{2}=n^{2} \\
\nabla \cdot\left(a^{2} \nabla \phi\right)=0 .
\end{gathered}
$$

The eiconal equation (1.5) determines the phase and the transport equation (1.6) determines the amplitude. Using a perturbation method to solve the transport equation we may obtain the geometrical optics result for (1.2). This result breaks down in some regions where there are caustic or foci. Since the wave motion should be bounded in the whole space the breakdown of the gecmetrical optics result must be recognized as asymptotic phenomena associated with boundary layers. $\mathbb{A}$ boundary layer is a thin region in which the dependent variable of differential equation undergoes sharp changes. To study the asymptotic bebavior in the regions where geometrical optics result breaks down, the boundary layer method is useful and the theory is well developed.

In this paper, we consider two dimensional problems for equation (1.1). Our object is to obtain an asymptotic solution for (1.1) near a caustic. For convenience we infroduce $(\xi, \sigma)$ coordinates where $\xi$ measure the arc length along the caustic and $\sigma$ measure the arc length along the rays. We first obtain the equation of nonlinear geometrical optics and discuss the boundary layer of the solution of (1.1). Then we introduce stretching transformation to obtain nonlinear Airy equation from (1.1). We follow the linear theory to discuss the asymptotic behavior of its solution. Finally we determine the asymptotic behavior of an incident beam after passing through the eaustic region. We find that the incident beam undergoes a phase shift by an amount of $\frac{\pi}{2}$ as it passes through the caustic and becomes an outgoing beam.

\section{Boundary layer of the nonlinear caustic}

To study how the field passes through a nonlinear caustic we consider the nonlinear reduced wave equation

$$
\nabla^{2} u+k^{2} n^{2}\left(|u|^{2}\right) u=0
$$

near the caustic region.

The rays for the geometrical optics solution of (2.1) are assumed to have a smooth envelope. This envelope is called a nonlinear caustic. The equation of the caustic is assumed to be

$$
X=\vec{R}(\xi)
$$

where $\xi$ is arc length on the caustic. The rays of the problem are the tangent lines to the caustic and are given as

$$
\vec{r}(\xi, \sigma)=\vec{R}(\xi)+(\sigma-\xi) \vec{R}^{\prime}(\xi)
$$


where $\sigma$ is arc length on the rays and $\vec{R}^{\prime}(\xi)=\vec{e}_{1}$ is a unit vector tangent to the caustic. To discuss the solution of (2.1) near the caustic it is convenient to write (2.1) in terms of $(\xi, \sigma)$ coor dinates. Differentiating (2.3) gives

$$
d \vec{r}=\vec{e}_{1} d \sigma+\frac{\sigma-\xi}{\rho} \vec{e}_{2} d \xi
$$

where $\vec{e}_{2}=\rho \frac{d \vec{e}_{1}}{d \xi}$ and $\rho(\xi)$ is the radius of curvature of the caustic. Hence we have

$$
\nabla^{2} u=\frac{1}{\xi-\sigma} \frac{\partial}{\partial \sigma}\left[(\xi-\sigma) \frac{\partial u}{\partial \sigma}\right]+\frac{\rho}{\xi-\sigma} \frac{\partial}{\partial \xi}\left[\frac{\rho}{\xi-\sigma} \frac{\partial u}{\partial \xi}\right]
$$

and (2.1) becomes

$$
\frac{1}{\xi-\sigma} \frac{\partial}{\partial \sigma}\left[(\xi-\sigma) \frac{\partial u}{\partial \sigma}\right]+\frac{\rho}{\xi-\sigma} \frac{\partial}{\partial \xi}\left[\frac{\rho}{\xi-\sigma} \frac{\partial u}{\partial \xi}\right]+k^{2} n^{2}\left(|u|^{2}\right) u=0 .
$$

Assuming $n^{2}\left(|u|^{2}\right)=1+n_{1}|u|^{2}+n_{2}|u|^{4}+\cdots$ and

$$
u=\frac{1}{\sqrt{k}} w e^{i k \sigma}
$$

we have

$$
\begin{aligned}
& i k\left[2 \frac{\partial w}{\partial \sigma}-\frac{w}{\xi-\sigma}\right]+\frac{1}{\xi-\sigma} \frac{\partial}{\partial \sigma}\left[(\xi-\sigma) \frac{\partial w}{\partial \sigma}\right]+\frac{\rho}{\xi-\sigma} \frac{\partial}{\partial \xi}\left[\frac{\rho}{\xi-\sigma} \frac{\partial w}{\partial \xi}\right] \\
& \quad+k n_{1}|w|^{2} w+n_{2}|w|^{4} w+\cdots=0 .
\end{aligned}
$$

Equation (2.8) can be expressed as equations for $a$ and $\phi$ by letting

$$
w=a e^{i \phi}
$$

where $a$ and $\phi$ are real.

Then

$$
\frac{\partial w}{\partial \sigma}=\frac{\partial a}{\partial \sigma} e^{i \phi}+i \frac{\partial \phi}{\partial \sigma} a e^{i \phi}
$$

and

$$
\begin{gathered}
i k\left[2 \frac{w}{\partial \sigma}-\frac{w}{\xi-\sigma}\right]=\left[i k\left(2 \frac{\partial a}{\partial \sigma}-\frac{a}{\xi-\sigma}\right)-2 a k \frac{\partial \phi}{\partial \sigma}\right] e^{i \phi} \\
\frac{\partial}{\partial \sigma}\left[(\xi-\sigma) \frac{\partial w}{\partial \sigma}\right]=\frac{\partial}{\partial \sigma}\left[\frac{\partial a}{\partial \sigma}(\xi-\sigma) e^{i \phi}+i \frac{\partial \phi}{\partial \sigma} e^{i \phi}(\xi-\sigma)\right] \\
=i\left[(\xi-\sigma) \frac{\partial^{2} \phi}{\partial \sigma^{2}} a+2 \frac{\partial \phi}{\partial \sigma} \frac{\partial a}{\partial \sigma}(\xi-\sigma)-\frac{\partial \phi}{\partial \sigma} a\right] e^{i \phi} \\
+\left\{\frac{\partial}{\partial \sigma}\left[(\xi-\sigma) \frac{\partial a}{\partial \sigma}\right]-\left(\frac{\partial \phi}{\partial \sigma}\right)^{2} a(\xi-\sigma)\right\} e^{i \phi}
\end{gathered}
$$




$$
\begin{aligned}
\frac{\partial}{\partial \xi}\left[\frac{\rho}{\xi-\sigma} \frac{\partial w}{\partial \xi}\right]= & \frac{\partial}{\partial \xi}\left[\frac{\rho}{\xi-\sigma}\left(\frac{\partial a}{\partial \xi} e^{i \phi}+i \frac{\partial \phi}{\partial \xi} a e^{i \phi}\right)\right] \\
= & e^{i \phi} \frac{\partial}{\partial \xi}\left[\frac{\rho}{\xi-\sigma} \frac{\partial a}{\partial \xi}\right]-e^{i \phi}\left(\frac{\partial \phi}{\partial \xi}\right)^{2} a \frac{\rho}{\xi-\sigma} \\
& +i e^{i \phi}\left[2 \frac{\rho}{\xi-\sigma} \frac{\partial a}{\partial \xi} \frac{\partial \phi}{\partial \xi}+a \frac{\partial \phi}{\partial \xi} \frac{\partial}{\partial \xi}\left(\frac{\rho}{\xi-\sigma}\right)+\frac{\rho}{\xi-\sigma} a \frac{\partial^{2} \phi}{\partial \xi^{2}}\right] .
\end{aligned}
$$

Therefore, we obtain on factoring out $e^{i \phi}$

$$
\begin{aligned}
& i\left\{k\left(2 \frac{\partial a}{\partial \sigma}-\frac{a}{\xi-\sigma}\right)+2 \frac{\partial a}{\partial \sigma} \frac{\partial \phi}{\partial \sigma}-\frac{a}{\xi-\sigma} \frac{\partial \phi}{\partial \sigma}+a \frac{\partial^{2} \phi}{\partial \sigma^{2}}+2 \frac{\rho^{2}}{(\xi-\sigma)^{2}} \frac{\partial a}{\partial \xi} \frac{\partial \phi}{\partial \xi}\right. \\
& \left.+\frac{\partial \phi}{\partial \xi} \frac{\rho}{\xi-\sigma} a \frac{\partial}{\partial \xi}\left(\frac{\rho}{\xi-\sigma}\right)+\frac{\rho^{2}}{(\xi-\sigma)^{2}} a \frac{\partial^{2} \phi}{\partial \xi^{2}}\right\}+\left\{\frac{1}{\xi-\sigma} \frac{\partial}{\partial \sigma}\left[(\xi-\sigma) \frac{\partial a}{\partial \sigma}\right]\right. \\
& +\frac{\rho}{\xi-\sigma} \frac{\partial}{\partial \xi}\left[\frac{\rho}{\xi-\sigma} \frac{\partial a}{\partial \xi}\right]-a\left(\frac{\partial \phi}{\partial \sigma}\right)^{2}-\frac{\rho^{2}}{(\xi-\sigma)^{2}} a\left(\frac{\partial \phi}{\partial \xi}\right)^{2} \\
& \left.-2 k a \frac{\partial \phi}{\partial \sigma}+k n_{1} a^{3}+n_{2} a^{5}+\cdots\right\}=0 .
\end{aligned}
$$

Equation real and imaginary parts to zero, we have

$$
\begin{aligned}
& k\left(2 \frac{\partial a}{\partial \sigma}-\frac{a}{\xi-\sigma}\right)+2\left[\frac{\partial a}{\partial \sigma} \frac{\partial \phi}{\partial \sigma}+\frac{\rho^{2}}{(\xi-\sigma)^{2}} \frac{\partial a}{\partial \xi} \frac{\partial \phi}{\partial \xi}\right] \\
& +a\left[\frac{\partial^{2} \phi}{\partial \sigma^{2}}-\frac{1}{\xi-\sigma} \frac{\partial \phi}{\partial \sigma}+\frac{\rho}{\xi-\sigma} \frac{\partial}{\partial \xi}\left(\frac{\rho}{\xi-\sigma}\right) \frac{\partial \phi}{\partial \xi}\right. \\
& \left.+\frac{\rho^{2}}{(\xi-\sigma)^{2}} \frac{\partial^{2} \phi}{\partial \xi^{2}}\right]=0
\end{aligned}
$$

and

$$
\begin{aligned}
& \frac{1}{\xi-\sigma} \frac{\partial}{\partial \sigma}\left[(\xi-\sigma) \frac{\partial a}{\partial \sigma}\right]+\frac{\rho}{\xi-\sigma} \frac{\partial}{\partial \xi}\left[\frac{\rho}{\xi-\sigma} \frac{\partial a}{\partial \xi}\right]-a \frac{\partial^{2} \phi}{\partial \sigma^{2}}-a \frac{\rho^{2}}{(\xi-\sigma)^{2}} \frac{\partial^{2} \phi}{\partial \xi^{2}} \\
& -2 a k \frac{\partial \phi}{\partial \sigma}+k n_{1} a^{3}+n_{2} a^{5}+\cdots=0 .
\end{aligned}
$$

(2.15) and (2.16) can be written as

$$
k\left[2 \frac{\partial a}{\partial \sigma}-\frac{a}{\xi-\sigma}\right]+2 \nabla a \cdot \nabla \phi+a \nabla^{2} \phi=0
$$

and

$$
\nabla^{2} a-a(\nabla \phi)^{2}-2 k a \frac{\partial \phi}{\partial \sigma}+k n_{1} a^{3}+k n_{2} a^{5}+\cdots=0 .
$$

If we neglect the diffraction term $\nabla^{2} a$ and higher order nonlinearities we obtain

$$
2 k \frac{\partial \phi}{\partial \sigma}+(\nabla \phi)^{2}=k n_{1} a^{2}
$$


and

$$
k\left(2 \frac{\partial \phi}{\partial \sigma}-\frac{a}{\xi-\sigma}\right)+2 \nabla a \cdot \nabla \phi+a \nabla^{2} \phi=0
$$

as the "equations of nonliear geometrical optics."

To see if we can find any beam-like solutions of these equations we stretch variables in the $\xi$-direction and thus emphasize a particular ray, say $\xi=0$. Then near $\xi=0$ we set

$$
\xi=k^{-r} \gamma
$$

so that $\frac{\partial}{\partial \xi}=k^{r} \frac{\partial}{\partial \gamma}$. We have

$$
\begin{aligned}
(\nabla \phi)^{2} & =\frac{\rho^{2}}{(\xi-\sigma)^{2}}\left(\frac{\partial \phi}{\partial \xi}\right)^{2}+\left(\frac{\partial \phi}{\partial \sigma}\right)^{2} \\
& =k^{2 r} \frac{\rho^{2}}{\sigma^{2}}\left(\frac{\partial \phi}{\partial \gamma}\right)^{2}+\left(\frac{\partial \phi}{\partial \sigma}\right)^{2}
\end{aligned}
$$

So we must set $r=\frac{1}{2}$ and (2.19) becomes

$$
2 \frac{\partial \phi}{\partial \sigma}+\frac{\rho^{2}}{\sigma^{2}}\left(\frac{\partial \phi}{\partial \gamma}\right)^{2}=n_{1} a^{2} ; \rho=\rho(0)
$$

to leading oder.

Also (2.20) becomes

$$
2 \frac{\partial a}{\partial \sigma}+\frac{a}{\sigma}+\frac{2 \rho^{2}}{\sigma^{2}} \frac{\partial a}{\partial \gamma} \frac{\partial \phi}{\partial \gamma}+a \frac{\rho^{2}}{\sigma^{2}} \frac{\partial^{2} \phi}{\partial \gamma^{2}}=0 .
$$

A simple solution of (2.23)-(2.24) may be found by assuming that

$$
a=a(\sigma) ; \phi=\phi(\sigma) \text {. }
$$

Then (2.23) becomes

$$
2 \frac{\partial \phi}{\partial \sigma}=n_{1} a^{2}
$$

and (2.24) becomes

$$
2 \frac{\partial a}{\partial \sigma}+\frac{a}{\sigma}=0
$$

Thus we have

$$
a=\frac{A}{\sqrt{\sigma}}
$$

and

$$
\phi=\frac{n_{1} A^{2}}{2} \log \sigma .
$$


Even if we retain the diffraction term $\nabla^{2} a$ in the equation, under the above stretching we still have the above solution (2.28). We note that this solution does not have the form of a beam. In order to get a beam solution of (2.23)-(2.24) we set

$$
a=\frac{A(\gamma)}{\sqrt{\sigma}}
$$

in (2.24). Since

$$
2 \frac{\partial a}{\partial \sigma}+\frac{a}{\sigma}=2 \frac{\partial}{\partial \sigma}\left(\frac{A(\gamma)}{\sqrt{\sigma}}\right)+\frac{1}{\sigma} \frac{A(\gamma)}{\sqrt{\sigma}}=0
$$

we have

$$
2 \frac{\rho^{2}}{\sigma^{2}} \frac{A^{\prime}(\gamma)}{\sqrt{\sigma}} \frac{\partial \phi}{\partial \gamma}+\frac{A(\gamma)}{\sqrt{\sigma}} \frac{\rho^{2}}{\sigma^{2}} \frac{\partial^{2} \phi}{\partial \gamma^{2}}=0
$$

This gives

$$
2 A^{\prime}(\gamma) \frac{\partial \phi}{\partial \gamma}+A(\gamma) \frac{\partial^{2} \phi}{\partial \gamma^{2}}=0
$$

or

$$
\frac{\partial}{\partial \gamma}\left[A^{2}(\gamma) \frac{\partial \phi}{\partial \gamma}\right]=0
$$

so that

$$
A^{2}(\gamma) \frac{\partial \phi}{\partial \gamma}=B(\sigma)
$$

where $B(\sigma)$ is arbitrary. From (2.23) we now have

$$
2 \frac{\partial \phi}{\partial \sigma}+\frac{\rho^{2}}{\sigma^{2}} \frac{B^{2}(\sigma)}{A^{4}(\gamma)}=n_{1} \frac{A^{2}(\gamma)}{\sigma}
$$

and we may solve for $\phi$ as

$$
\phi=\frac{n_{1}}{2} A^{2}(\gamma) \log \sigma-\frac{\rho^{2}}{2 A^{4}(\gamma)} \int^{\sigma} \frac{B^{2}(\sigma)}{\sigma^{2}} d \sigma+H(\gamma)
$$

we must choose $H(\gamma)$ to get consistency. Differentiating (2.36) with respect to $\gamma$, we have

$$
2 \frac{\partial^{2} \phi}{\partial \gamma \partial \sigma}-\frac{4 \rho^{2} A^{\prime}(\gamma)}{A^{5}(\gamma)} \frac{B^{2}(\sigma)}{\sigma^{2}}=2 n_{1} A(\gamma) A^{\prime}(\gamma) \frac{1}{\sigma}
$$

and this gives

$$
2 B^{\prime}(\sigma) \sigma-\frac{4 \rho^{2} A^{\prime}(\gamma)}{A^{3}(\gamma)} \frac{B^{2}(\sigma)}{\sigma}=2 n_{1} A^{3}(\gamma) A^{\prime}(\gamma) .
$$

It appears that we must set

$$
A^{\prime}(\gamma)=0 ; B^{\prime}(\sigma)=0
$$

so that $A(\gamma)$ is independent of $\gamma$. 
The above result tells us that it seems hard to construct a beam solution for (2.23)(2.24) by above method. But by using the perturbation method we are able to obtain a beam-like solution of (2.1) for that system of rays discussed above. To show this, we introduce the caustic coordinate system $(\xi, \sigma)$ and express (2.1) in the form (2.6). Then we set

$$
u=\frac{1}{\sqrt{k}} a e^{i k \phi}
$$

with

$$
a=\sum_{j=0}^{\infty} a_{j} k^{-j} ; \phi=\sum_{j=0}^{\infty} \phi_{j} k^{-j}
$$

we have

$$
\left(\frac{\partial \phi_{0}}{\partial \sigma}\right)^{2}+\frac{\rho^{2}}{(\xi-\sigma)^{2}}\left(\frac{\partial \phi_{0}}{\partial \xi}\right)^{2}=1
$$

and we choose

$$
\phi_{0}=\sigma
$$

to obtain the required system of rays. The equation for $a_{0}$ is

$$
2 \frac{\partial a_{0}}{\partial \sigma}-\frac{a_{0}}{\xi-\sigma}=0
$$

and we conclude that

$$
a_{0}=\frac{g(\xi)}{\sqrt{\xi-\sigma}}
$$

where $g(\xi)$ is a given function.

If we assume that $g(\xi)=e^{-\xi / \alpha^{2}}$ then (2.46) looks like a beam.

For $\phi_{1}$, we obtain

$$
2 \frac{\partial \phi_{1}}{\partial \sigma}=n_{1} \alpha_{0}^{2}
$$

so that

$$
\phi_{1}=-\frac{n_{1}}{2} g^{2}(\xi) \log (\xi-\sigma)
$$

For $a_{1}$, we have

$$
2 \frac{\partial a_{1}}{\partial \sigma}-\frac{a_{1}}{\xi-\sigma}=-\frac{3 \rho^{2} n_{1} g^{3}(\xi)}{2(\xi-\sigma)^{9 / 2}}+\cdots
$$

where we have retained only the most singular term near the caustic where $\sigma=\xi$. Them

$$
\begin{aligned}
a_{1} & =-\frac{n_{1}}{4} \frac{\rho^{2} g^{3}(\xi)}{(\xi-\sigma)^{7 / 2}}+\cdots \\
& =-\frac{n_{1}}{4} \frac{\rho^{2} g^{2}(\xi)}{(\xi-\sigma)^{3}} a_{0}+\cdots
\end{aligned}
$$


The asymptotic series for $a$ becomes disordered when $\frac{a_{1}}{k} \approx a_{0}$ and this yields

$$
-\frac{n_{1} \rho^{2} g^{2}(\xi)}{4 k(\xi-\sigma)^{3}} \approx 1
$$

So that

$$
\xi-\sigma \approx \frac{c n_{1}^{1 / 3} \rho^{2 / 3} g^{2 / 3}(\xi)}{k^{1 / 3}} ; \quad c=\text { constant }
$$

That is, when $\xi-\sigma=O\left(k^{-1 / 3}\right)$ the caustic regien begins. Since $a_{0}=\frac{g(\xi)}{\sqrt{\xi-\sigma}}$ we see that $a_{0}=O\left(k^{1 / 6}\right)$ near the caustie. For the linear case, to obtain the standard geometrical optics expansien, we set

$$
u=w e^{i k \phi}
$$

in (2.1) where $n_{1}=n_{2}=0$ and $w$ is expanded in powers of $(i k)^{-1}$. Then

$$
w_{0}=\frac{g(\xi)}{\sqrt{\xi-\sigma}} ; w_{1}=\frac{g(\xi) \rho^{2}}{(\xi-\sigma)^{7 / 2}}=w_{0} \frac{\rho^{2}}{(\xi-\sigma)^{3}}
$$

near the caustic. Thus $\frac{w_{1}}{i k} \approx w_{0}$ yields

$$
\xi-\sigma=\approx \hat{c} \frac{\rho^{2 / 3}}{k^{1 / 3}}, \hat{c}=\text { constant }
$$

This shows that the caustic regions determined from (2.52) and (2.55) differ by the factor $n_{1}^{1 / 3} g^{2 / 3}(\xi)$. As $n_{1}$ and $g(\xi)$ are increased, the self focusing effect becomes enhanced and the caustic becomes more sharply defined. This should be compared with the expression for the focal length and its relation to the amplitude and nonlinear refraction term $n_{1}$.

We now study the field in the neighborhood of the caustic. We stretch the dependent and independent variables as

$$
\xi-\sigma=k^{-r} \eta ; \xi=\lambda ; \cdot v=k^{s} v
$$

Then (2.8) becomes

$$
\begin{aligned}
& -i k^{1+r}\left[2 \frac{\partial v}{\partial \eta}+\frac{v}{\eta}\right]+\frac{k^{2 r}}{\eta} \frac{\partial}{\partial \eta}\left[\eta \frac{\partial v}{\partial \eta}\right]+\frac{k^{r} \rho}{\eta}\left[\frac{\partial}{\partial \lambda}+k^{r} \frac{\partial}{\partial \eta}\right]\left[\frac{k^{r} \rho}{\eta}\left(\frac{\partial v}{\partial \lambda}+k^{r} \frac{\partial v}{\partial \eta}\right)\right] \\
& +k^{1+2 s} n_{1}|v|^{2} v+k^{4 s} n_{2}|v|^{4} v+\cdots=0 .
\end{aligned}
$$

In order to simplify the above equation, we only keep the significant terms in (2.57). According to the linear theory, the coefficient of $k^{1+r}$ should be retained. We also have to 
retain at least one second derivative term in (2.57), this term comes from the coefficient of $k^{4 r}$. To retain a nonlinear effect we must set

$$
1+r=4 r=1+2 s
$$

so that

$$
r=\frac{1}{3} \quad ; \quad s=\frac{1}{6} .
$$

The leading order boundary layer equation becomes

$$
-i\left[2 \frac{\partial v}{\partial \eta}+\frac{v}{\eta}\right]+\frac{\rho^{2}}{\eta} \frac{\partial}{\partial \eta}\left[\frac{1}{\eta} \frac{\partial v}{\partial \eta}\right]+n_{1}|v|^{2} v=0 .
$$

This equation may be simplified by letting

$$
v=e^{\frac{i \eta^{3}}{3 p^{2}}} V(z)
$$

where

$$
z=\frac{\eta^{2}}{\left(4 \rho^{4}\right)^{1 / 3}} .
$$

This yields a nonlinear Airy equation

$$
V^{\prime \prime}(z)+z V(z)+\frac{n_{1} \rho^{2 / 3}}{4^{1 / 3}}|V|^{2} V(z)=0
$$

or

$$
V^{\prime \prime}+\left[z+\frac{n_{1} \rho^{2 / 3}}{4^{1 / 3}}|V|^{2}\right] V=0 .
$$

The above equation shows that the turning point for the Airy equation is approximately given by

$$
z+\frac{n_{1} \rho^{2 / 3}}{4^{1 / 3}}|V|^{2}=0
$$

If we set

$$
|V(z)|^{2}=\frac{g^{2}(\xi)}{(\xi-\sigma) k^{1 / 3}}
$$

we find that

$$
\begin{aligned}
& \frac{\eta^{2}}{\left(4 \rho^{4}\right)^{1 / 3}}+\frac{n_{1} \rho^{2 / 3}}{4^{1 / 3}}|V(z)|^{2} \\
= & \frac{(\xi-\sigma)^{2}}{\left(4 \rho^{4}\right)^{1 / 3}} k^{2 / 3}+\frac{n_{1} \rho^{2 / 3}}{4^{1 / 3}} g^{2}(\xi)(\xi-\sigma)^{-1} k^{-1 / 3}=0 .
\end{aligned}
$$

Thus

$$
(\xi-\sigma)^{3}=-\frac{n_{1} \rho^{2} g^{2}(\xi)}{k}
$$


which agrees with (2.52) and yields

$$
\xi-\sigma=-n_{1}^{1 / 3} \rho^{2 / 3} k^{-1 / 3} g^{2 / 3}(\xi) .
$$

Roughly speaking this may be thought of as a shift in the location of the caustic curve from $\xi=\sigma$ in the linear case to

$$
\sigma=\xi+n_{1}^{1 / 3} \rho^{2 / 3} k^{-1 / 3} g^{2 / 3}(\xi)
$$

in the nonlinear case. The direction of the shift depends on the sign of $n_{1}$.

With $z$ replaced by $-z,(2.63)$ becomes

$$
\widehat{V}^{\prime \prime}(z)-z \widehat{V}(z)+\frac{n_{1} \rho^{2 / 3}}{4^{1 / 3}}|\widehat{V}(z)|^{2} \widehat{V}(z)=0
$$

where

$$
\widehat{V}(z)=V(-z)
$$

If we assume that $\widehat{V}$ is real and set

$$
\widehat{V}(z)=\sqrt{2}\left(n_{1} \rho^{2 / 3} 4^{-1 / 3}\right)^{-1 / 2} W(z) ; \quad n_{1}>0
$$

then (2.71) can be simplified as

$$
W^{\prime \prime}(z)-z W(z)+2 W^{3}(z)=0 .
$$

This nonlinear differential equation is the second equation of painleve ${ }^{[1]}$. The solution of (2.74) can not be represented by elementary functions. We need to define a new function for its solution which is called second painlevé transcendent. It has been shown that the solution of (2.74) can be given by the solution of the linear integral equation

$$
K(\xi, n)=F\left(\frac{\xi+n}{2}\right)-\int_{\xi}^{\infty} K(\xi, \psi) F\left(\frac{\psi+\phi}{2}\right)\left(\frac{\phi+n}{2}\right) d \psi d \phi \quad \eta \geq \xi
$$

where $F(\xi)$ is the solution of

$$
F^{\prime \prime}(\xi)-\xi F(\xi)=c, \quad c=\text { constant }
$$

If $c=0$ the solution of (2.76) that vanish as $\xi \rightarrow \infty$ are multiple of the Airy function

$$
F(\xi)=\lambda A_{i}(\xi)
$$

A detailed discussion of the above can be found in Ablowitz and Segur. [2]

\section{The asymptotic expanssion near a nonlinear caustic}


To get an approximate solution of (2.74) we use the result of Miles ${ }^{[3]}$. There is a solution of (2.74) with the behavior

$$
\begin{aligned}
W(z) & \approx \hat{a} A_{i}(z) \quad ; \hat{\omega}=\text { constant } \\
& \approx \frac{1}{2} \pi^{-1 / 2} \hat{\alpha} z^{-1 / 4} \exp \left[-\frac{2}{3} z^{3 / 2}\right] ; \quad z \rightarrow \infty
\end{aligned}
$$

with the property

$$
|W(z)|<\infty \quad ; \quad-\infty<z<\infty
$$

In terms of $V(z)$ we have

$$
\begin{aligned}
V(z) & =\left[\frac{n_{1}}{2} \rho^{2 / 3_{4}} 4^{-1 / 3}\right]^{-1 / 2} W(-z) \\
& \approx \hat{a}\left[\frac{n_{1}}{2} \rho^{2 / 3} \mathbb{4}^{-1 / 3}\right]^{-1 / 2} A_{i}(-z) \\
& \approx \hat{a}\left[2 n_{1} \pi \rho^{2 / 3} \mathbb{4}^{-1 / 3}\right]^{-1 / 2}(-z)^{-1 / 4} \exp \left[-\frac{2}{3}(-z)^{3 / 2}\right] ; \quad z \rightarrow \infty
\end{aligned}
$$

Further, as $z \rightarrow \infty$ the asymptotic behavior of $V(z)$ is

$$
V(z) \approx \alpha z^{-1 / 4} \sin \left[\frac{2}{3} z^{3 / 2}+\frac{3}{4} \alpha^{2} \log z+\theta\right] ; \quad z \rightarrow \infty
$$

where $\alpha, \theta$ are constants.

This expression for $V(z)$ must be related to the result (2.41). In view of (2.62) we have

$$
\begin{aligned}
V \approx & \alpha \frac{\left(4 \rho^{4}\right)^{1 / 12} k^{-1 / 6}}{(\xi-\sigma)^{1 / 2}} \sin \left[\frac{1}{3} \frac{\eta^{3}}{\rho^{2}}+\frac{3}{4} \alpha^{2} \log \frac{\eta^{2}}{\left(4 \rho^{4}\right)^{1 / 3}}+\theta\right] \\
= & \frac{1}{2} \frac{\alpha\left(4 \rho^{4}\right)^{1 / 12} k^{-1 / 6}}{(\xi-\sigma)^{1 / 2}} e^{+i\left[\frac{\eta}{3 \rho^{2}}+\frac{3}{4} \alpha^{2} \log \eta^{2} /\left(4 \rho^{4}\right)^{1 / 3}+\theta-\frac{\pi}{2}\right]} \\
& +\frac{1}{2} \frac{\alpha\left(4 \rho^{4}\right)^{1 / 12} k^{-1 / 6}}{(\xi-\sigma)^{1 / 2}} e^{-i\left[\frac{\eta}{3 \rho^{2}}+\frac{3}{4} \alpha^{2} \log \eta^{2} /\left(4 \rho^{4}\right)^{1 / 3}+\theta-\frac{\pi}{2}\right]} .
\end{aligned}
$$

Since $W=k^{1 / 6} v=k^{1 / 6} e^{\frac{i \eta^{3}}{3 \rho^{2}}} V(z)$, we have $W=O(1)$ in $k$. From (3.5) we may say that it contains two fields. One is incident field and the other one is outgoing field. Two fields coexist in the region near the caustic. Because the equation is nonlinear, anyone of two fields may not be a solution of the equation. We can not discuss them separately. From the view point of geometrical optics we can obtain the geometrical optics solutions of equation (2.63) in two sides of the caustic. From the given initial condition the incident geometrical optics result can be easily determined. Our problem is to try to determine the geometrical opties result for the outgoing field. The result of (3.5) is not useful to 
us, because it is not possible to match these fields with our geometrical optices results one by one, so that we have to discuss (2.63) directly.

To solve (2.63) approximately, we set

$$
z=\frac{1}{\varepsilon^{s}} x ; \quad V=\varepsilon^{r} \bar{v}
$$

where $\varepsilon=O\left[k^{-1}\right]$. Substituting (3.6) into (2.63) we have

$$
\varepsilon^{r+2 s} \bar{v}^{\prime \prime}+\varepsilon^{r-s} 2 \bar{v}+A \varepsilon^{3 r}|\bar{v}|^{2} \bar{v}=0
$$

where $A=\frac{n_{1} \rho^{2 / 3}}{4^{1 / 3}}$.

Eqation (3.7) can be simplified as

$$
\bar{v}^{\prime \prime}+\varepsilon^{-3 s} x \bar{v}+A \varepsilon^{2(r-s)}|\bar{v}|^{2} \bar{v}=0 .
$$

Let

$$
\bar{v}=a e^{i \phi / \varepsilon}
$$

where $a, \phi$ are real. (3.8) becomes

$$
\left(\varepsilon^{-3 s} \mathfrak{W}-\varepsilon^{-2} \phi^{2}\right) a+i \varepsilon\left(2 \phi^{\prime} a^{\prime}+a \phi^{\prime \prime}\right)+a^{\prime \prime}+A \varepsilon^{2(r-s)} a^{3}=0 .
$$

In order to keep both $x$ and $\phi^{\prime 2}$ in (3.10) we set $3 s=2$. Eqation (3.10) becomes

$$
\varepsilon^{-2}\left(x-\phi^{\prime 2}\right) a+i \varepsilon^{-1}\left(2 \phi^{\prime} a^{\prime}+a \phi^{\prime \prime}\right)+a^{\prime \prime}+A \varepsilon^{2\left(r-\frac{2}{3}\right)} a^{3}=0 .
$$

Equating real and imaginary parts of (3.11) to zero, we have

$$
\varepsilon^{-2}\left(x-\phi^{\prime 2}\right) a+a^{\prime \prime}+A \varepsilon^{2 r-\frac{4}{3}} a^{3}=0
$$

and

$$
2 \phi^{\prime} a^{\prime}+a \phi^{\prime \prime}=0
$$

We expand $\phi$ and $a$ as

$$
\phi=\sum_{j=0}^{\infty} \phi_{j} \varepsilon^{j}
$$

and

$$
a=\sum_{j=0}^{\infty} a_{j} \varepsilon^{j} .
$$

Inserting these expressions into (3.12) we obtain

$$
\left(x-\phi_{0}^{\prime 2}\right) a_{0}+\varepsilon\left(2 \phi_{0}^{\prime} \phi_{1}^{\prime}\right) a_{0}+A \varepsilon^{2 r+\frac{2}{3}} a_{0}^{3}+\varepsilon^{2} a_{0}^{\prime \prime}+\varepsilon^{2}\left(2 \phi_{0}^{\prime} \phi_{1}^{\prime} a_{1}\right)=0 .
$$


According to (3.5) the field is equal to $O\left[k^{-\frac{1}{6}}\right.$. $]$ so we choose $r=1 / 6$ and equating the coefficient of each power of $\varepsilon$ to zero, we have

$$
\phi_{0}^{\prime 2}=x ; \quad x>0
$$

and

$$
2 \phi_{0}^{\prime} \phi_{1}^{\prime}+A a_{0}^{2}=0
$$

Solving (3.17) we have

$$
\begin{gathered}
\phi_{i 0}=-\frac{2}{3} x^{\frac{3}{2}}=-\frac{2}{3} z^{\frac{3}{2}} \varepsilon ; \quad z=\frac{(\xi-\sigma)^{2} k^{2 / 3}}{\left(4 \rho^{4}\right)^{1 / 3}} \\
\phi_{00}=\frac{2}{3} x^{\frac{3}{2}}=\frac{2}{3} z^{\frac{3}{2}} \varepsilon ; \quad z=\frac{(\sigma-\xi)^{2} k^{2 / 3}}{\left(4 \rho^{4}\right)^{1 / 3}}
\end{gathered}
$$

From (3.13) we have

$$
2 \phi_{0}^{\prime} a_{0}^{\prime}+a_{0} \phi_{0}^{\prime \prime}=0 .
$$

Solving (3.21) and (3.18) we obtain

$$
a_{i 0}=\frac{C_{i}(\xi)}{x^{1 / 4}}=\frac{\varepsilon^{-1 / 6}}{z^{1 / 4}} C_{i}(\xi)
$$

where $C_{i}(\xi)$ is are arbitrary function of $\xi$, and

$$
\phi_{i 1}=\frac{A C_{i}^{2}(\xi)}{2} \log z+\alpha_{1}(\xi)
$$

where $\alpha_{1}(\xi)$ is am arbitrary function.

If we repeat the same calculation for $\phi_{00}=\frac{2}{3} x^{\frac{3}{2}}$ we have

$$
a_{00}=\frac{C_{0}(\xi)}{x^{1 / 4}}=\frac{\varepsilon^{-1 / 6}}{z^{1 / 4}} C_{0}(\xi)
$$

and

$$
\phi_{01}=\frac{A C_{0}^{2}(\xi)}{2} \log z+\alpha_{2}(\xi)
$$

where $C_{0}(\xi)$ and $\alpha_{2}(\xi)$ are arbitrary functions.

Now we have two different asymptotic expansions for the solution of (2.63). One asymptotic result is obtained from (3.6), (3.9), (3.19), (3.22) and (3.23)

$$
V_{i}(z)=\frac{C_{i}(\xi)}{z^{1 / 4}} e^{-i \frac{2}{3} z^{\frac{3}{2}}+i \frac{A C_{i}^{2}(\xi)}{2} \log z+i \alpha_{1}(\xi)}
$$


According to (2.41), (2.44), (2.46) and (2.48) we have the incident field of the geometrical optics

$$
u_{i}=\frac{1}{\sqrt{k}} \frac{g(\xi)}{\sqrt{\xi-\sigma)}} e^{i k \sigma-i \frac{n_{1}}{2} g^{2}(\xi) \log (\xi-\sigma)} ; \quad \xi>\sigma .
$$

using (3.26), (2.7), (2.56) and (2.61) we also obatin the incident field near the caustic

$$
\hat{u}_{i}=\frac{1}{k^{1 / 3}} \frac{C_{i}(\xi)}{z^{1 / 4}} e^{i k \sigma+\frac{i A C_{i}^{2}(\xi)}{2} \log z+i \alpha_{1}(\xi)} .
$$

A second asymptotic result follows from (3.6), (3.9), (3.20), (3.24) and (3.25)

$$
V_{0}(z)=\frac{C_{0}(\xi)}{z^{1 / 4}} e^{i \frac{2}{3} z^{\frac{3}{2}}-i \frac{A C_{0}^{2}(\xi)}{2} \log z+i \alpha_{2}(\xi)}
$$

Since the ray structure is similar for both fields, the outgoing field of geometrical optics can be written as

$$
u_{0}=\frac{1}{\sqrt{k}} \frac{g_{0}(\xi)}{\sqrt{\sigma-\xi}} e^{i k \sigma-i \frac{n_{1}}{2} g_{0}^{2}(\xi) \log (\sigma-\xi)+i g_{1}(\xi)} ; \quad \sigma>\xi
$$

where $g_{0}, g_{1}$ have to be determined. Using (3.29), (2.7), (2.56) and (3.25), the outgoing field near the caustic can be written as

$$
\hat{\imath}_{0}=\frac{1}{k^{1 / 3}} \frac{C_{0}(\xi)}{z^{1 / 4}} e^{i \varepsilon_{0-i} \frac{A C_{0}^{2}(\xi)}{2} \log z+i \alpha_{2}(\xi)}
$$

We must specify the terms $C_{i}(\xi), C_{0}(\xi), g_{0}(\xi)$ and $g_{1}(\xi)$. To determine $C_{i}(\xi)$, we match the most significant term in (3.28) and the most significant term in (3.27) in the region $k>(\xi-\sigma)^{3} k>>1$ where we also have $k \sigma>>\log z$. The most significant term in (3.28) is

$$
\begin{aligned}
\hat{u}_{i} & \approx \frac{1}{k^{1 / 3}} \frac{C_{i}(\xi)}{z^{1 / 4}} e^{i k \sigma} e^{i \alpha_{1}(\xi)} \\
& =\frac{1}{\sqrt{k}} \frac{C_{i}(\xi)}{\sqrt{\xi-\sigma}} 2^{1 / 6} \rho^{1 / 3} e^{i k \sigma} e^{i \alpha_{1}(\xi)} .
\end{aligned}
$$

The most signficant term in (3.27) is

$$
u_{i} \approx \frac{1}{\sqrt{k}} \frac{g(\xi)}{\sqrt{\xi-\sigma}} e^{i k \sigma}
$$

comparing (3.32) and (3.33) we obtain

$$
C_{i}(\xi)=\frac{g(\xi)}{2^{1 / 6} \rho^{1 / 3}}
$$


and

$$
\alpha_{1}(\xi)=0
$$

Then (3.28) becomes

$$
\hat{\imath}_{i}=\frac{g(\xi)}{k^{1 / 3} 2^{1 / 6} \rho^{1 / 3}} z^{-1 / A_{1}} e^{i k \sigma+i \frac{\eta_{1}}{2} g^{2}(\xi) \log z} .
$$

Now we must determine $C_{0}(\xi), g_{0}(\xi)$ and $g_{1}(\xi)$. Because $\hat{\varkappa}_{i}$ is incident on the caustic $(\xi>\sigma)$ and $\hat{\vartheta}_{0}$ is outgoing from the caustic $(\sigma>\xi)$ it is not possible to match them to obatin $C_{0}(\xi)$. In order to solve our problem we consider the linearized version of equation (2.63) which is

$$
V^{\prime \prime}(z)+z V(z)=0 .
$$

Equation (3.37) has two linearly independent solutions $A_{i}(-z)$ and $B_{i}(-z)$ where $A_{i}(z)$ and $B_{i}(z)$ are the Airy functions. From the formula [4]

$$
A_{i}(z)=-\omega A_{i}(\omega z)-\omega^{2} A_{i}\left(\omega^{2} z\right)
$$

and

$$
B_{i}(z)=i \omega A_{i}(\omega z)-i \omega^{2} A_{i}\left(\omega^{2} z\right)
$$

where $\omega=e^{-2 \pi i / 3}$ we find that

$$
\begin{aligned}
\widehat{V}(z) & =a_{1}(\xi) \frac{1}{2 i \omega}\left[B_{i}(-z)-i A_{i}(-z)\right] \\
& =a_{1}(\xi) A_{i}(-\omega z) ; \quad \xi>\sigma
\end{aligned}
$$

is a solution of (3.37) where $a_{1}(\xi)$ is a function to be determined. Using the asymptotic formula.

$$
A_{i}(z) \approx \frac{1}{2 \sqrt{\pi}} z^{\frac{-1}{1}} e^{-\frac{2}{3} z^{3 / 2}} ;|\operatorname{Arg} z|<\pi,|z|>>1
$$

we can write (3.40) as

$$
\widehat{V}(z) \approx a_{1}(\varepsilon) \frac{1}{2 \sqrt{\pi}} z^{-\frac{1}{4}} e^{i \frac{\pi}{12}} e^{-i \frac{2}{3} z \frac{3}{2}} ; \quad \xi>\sigma .
$$

Because $z>>\mathbb{1}$ we have $z>>\log z$ and (3.26) becomes

$$
\begin{aligned}
V_{i}(z) & \approx C_{i}(\xi) z^{-\frac{1}{4}} e^{-i \frac{2}{3} z^{\frac{3}{2}}} \\
& \approx \frac{g(\xi)}{2^{1 / 6} \rho^{1 / 3}} z^{-\frac{1}{4}} e^{-i \frac{2}{3} z^{\frac{3}{2}}} ; \quad z>>1, \xi>\sigma .
\end{aligned}
$$

To specify $a_{1}(\xi)$ we compare (3.42) with (3.43) in the region $z>>1$, we have

$$
a_{1}(\xi)=\sqrt{\pi} 2^{5 / 6} \rho^{-1 / 3} g(\xi) e^{i \frac{\pi}{12}} .
$$


Because equations (2.63) and (3.37) in the $(\xi, \sigma)$ variables are unchanged if $(\xi-\sigma)$ is replaced by $(\sigma-\xi)$. Thus for $\sigma>\xi$ the solution of (3.37) has the form

$$
\begin{aligned}
& \quad \bar{V}(z)=a_{2}(\xi) \frac{-1}{2 i \omega^{2}}\left[i A_{i}(-z)+B_{i}(-z)\right] \\
& =a_{2}(\xi) A_{i}\left(-\omega^{2} z\right)
\end{aligned}
$$

where $a_{2}(\xi)$ has to be determined.

Using formula (3.41) we have

$$
\bar{V}(z) \approx a_{2}(\xi) \frac{1}{2 \sqrt{\pi}} z^{-\frac{1}{4}} e^{i \frac{\pi}{12}} e^{i \frac{2}{3} z^{\frac{3}{2}}} ; \quad z>>1
$$

By the same reason as for $V_{i}(z)$ from (3.29) we have

$$
V_{0} \approx C_{0}(\xi) z^{-\frac{1}{4}} e^{i \frac{2}{3} z^{\frac{3}{2}}} e^{i \alpha_{2}(\xi)}, \quad z>>1
$$

Comparing (3.46) with (3.47) we have

$$
a_{2}(\xi)=2 \sqrt{\pi} C_{0}(\xi) e^{i\left[\alpha_{2}(\xi)-\frac{\pi}{12}\right]} .
$$

Two linearly independent solutions of (3.37) become

$$
\widehat{V}(z)=\sqrt{\pi} 2^{5 / 6} \rho^{-1 / 3} g(\xi) e^{i \frac{\pi}{12}} A_{i}(-\omega z) ; \quad \xi>\sigma
$$

and

$$
\bar{V}(z)=2 \sqrt{\pi} C_{0}(\xi) e^{i\left[\alpha_{2}(\xi)-\frac{\pi}{12}\right]} A_{i}\left(-\omega^{2} z\right) ; \quad \sigma>\xi .
$$

To determine $C_{0}(\xi)$ and $\alpha_{2}(\xi)$, we require that the field below the caustic be exponentially small. In order to use this fact, an approximate expression for the distance along the normal line to the caustic, valid in the boundary layer region, will be found. In the system of rays (2.3), the wavefronts are the curves $\sigma=$ constant. They are orthogonal to the rays and the rays are tangent to the caustic. Thus, along the wavefront, distance is given as $d s=\frac{|\xi-\sigma|}{\rho} d \xi$. According to (2.56) the boundary layer region is determined by $\xi=\sigma+O\left(k^{-1 / 3}\right)$. Integrating $d s$ along the wavefront $\sigma=$ constant, in the boundary layer region, we obatin

$$
\begin{aligned}
s & \approx \frac{1}{2 \rho}(\xi-\sigma)^{2} \\
& =k^{-\frac{2}{3}} \frac{\eta^{2}}{2 \rho} \\
& =\left(\frac{\rho}{2}\right)^{1 / 3} k^{-\frac{2}{3}} z .
\end{aligned}
$$


where (2.56) and (2.62) were used. Within the boundary layer the variable $s$ yields a measure of distance along the normal line to the caustic, i.e. in the rays region. Negative values of $s$ correspond to points below the caustic. Using (3.51) we express (3.49) and (3.50) in terms of $\xi$ and $s$, we have

$$
\widehat{V}(z) \approx \sqrt{\pi} 2^{5 / 6} \rho^{-1 / 3} g(\xi) e^{i \frac{\pi}{12}} A_{i}\left[-\omega\left(\frac{2}{\rho}\right)^{\frac{1}{3}} k^{\frac{2}{3}} s\right]
$$

and

$$
\bar{V}(z) \approx 2 \sqrt{\pi} C_{0}(\xi) e^{i\left[\alpha_{2}(\xi)-\frac{\pi}{12}\right]} A_{i}\left[-\omega^{2}\left(\frac{2}{\rho}\right)^{\frac{1}{3}} k^{\frac{2}{3}} s\right] .
$$

We expect that $C_{0}(\xi)$ must be expressed in terms of $g(\xi)$. To establish this relationship we examine the total field $\hat{V}(z)+\bar{V}(z)$ in the boundary layer region. Because there is no distinction between $\xi>\sigma$ and $\sigma>\xi$ at a point $(\xi, s)$, the total field is gives as

$$
\begin{aligned}
\widehat{V}(z)+\bar{V}(z) \approx & \sqrt{\pi} 2^{5 / 6} \rho^{-1 / 3} g(\xi) e^{i \frac{\pi}{12}} A_{i}\left[-\omega\left(\frac{2}{\rho}\right)^{1 / 3} k^{2 / 3} s\right] \\
& +2 \sqrt{\pi} C_{0}(\xi) e^{i\left[\alpha_{2}(\xi)-\frac{\pi}{12}\right]} A_{i}\left[-\omega^{2}\left(\frac{2}{\rho}\right)^{1 / 3} k^{2 / 3} s\right] .
\end{aligned}
$$

In order to guarantee that the field is exponentially small below the caustic, we have to write the right hand side of (3.54) as a multiple of $A_{i}\left[-\left(\frac{2}{\rho}\right)^{1 / 3} k^{2 / 3} s\right]$ which decays as $s \rightarrow \infty$. Thus, the right hand side of (3.54) have to be written as

$$
\begin{aligned}
& \sqrt{\pi} 2^{5 / 6} \rho^{-1 / 3} g(\xi) e^{i \frac{\pi}{12}} A_{i}\left[-\omega\left(\frac{2}{\rho}\right)^{\frac{1}{3}} k^{\frac{2}{3}} s\right] \\
& +2 \sqrt{\pi} C_{0}(\xi) e^{i\left[\alpha_{2}(\xi)-\frac{\pi}{12}\right]} A_{i}\left[-\omega^{2}\left(\frac{2}{\rho}\right)^{\frac{1}{3}} k^{\frac{2}{3}} s\right] \\
& \left.=\frac{\sqrt{\pi} 2^{5 / 6} \rho^{1 / 3} g(\xi) e^{i \frac{\pi}{12}}}{-\omega}(-\omega) A_{i}\left[-\omega \frac{2}{\rho}\right)^{\frac{1}{3}} k^{\frac{2}{3}} s\right] \\
& +\frac{2 \sqrt{\pi} C_{0}(\xi) e^{i\left[\alpha_{2}(\xi)-\frac{\pi}{12}\right]}}{-\omega^{2}}\left(-\omega^{2}\right) A_{i}\left[-\omega^{2}\left(\frac{2}{\rho}\right)^{\left.\frac{1}{3} k^{\frac{2}{3}} s\right]}\right. \\
& =\sqrt{\pi} 2^{5 / 6} \rho^{-1 / 3} g(\xi) e^{i \frac{\pi}{12}} A_{i}\left[-\left(\frac{2}{\rho}\right)^{\frac{1}{3}} k^{\frac{2}{3}} s\right]
\end{aligned}
$$

which implies

$$
\frac{\sqrt{\pi} 2^{5 / 6} \rho^{-1 / 3} e^{i \frac{\pi}{12}} g(\xi)}{-\omega}=\frac{2 \pi C_{0}(\xi) e^{i\left[\alpha_{2}(\xi)-\frac{\pi}{12}\right]}}{-\omega^{2}}
$$

Solving (3.56) we have

$$
C_{0}(\xi)=\frac{g(\xi)}{2^{1 / 6} \rho^{1 / 3}} ; \quad \alpha_{2}(\xi)=-\frac{\pi}{2}
$$


Thus according to (3.57), the outgoing field near the caustic has the form

$$
\begin{aligned}
\hat{u}_{0} & \approx \frac{g(\xi)}{k^{1 / 3} 2^{1 / 6} \rho^{1 / 3}} z^{-\frac{1}{1}} e^{i k \sigma-i \frac{\pi}{2}} \\
& \approx \frac{g(\xi)}{\sqrt{k} \sqrt{\sigma-\xi}} e^{i k \sigma-i \frac{\pi}{2}} .
\end{aligned}
$$

where $k>>(\sigma-\xi)^{3} k>>1$.

To determine $g_{0}(\xi)$ and $g_{1}(\xi)$ in (3.30), we express (3.30) in the region $k>(\sigma-$ $\xi)^{3} k>>1$ where $k>>\log (\sigma-\xi)$ we have

$$
u_{0} \approx \frac{1}{\sqrt{k}} \frac{g_{0}(\xi)}{\sqrt{\sigma-\xi}} e^{i k_{0}+i g_{1}(\xi)} .
$$

To match (3.59) with (3.58), we have

$$
g_{0}(\xi)=g(\xi)
$$

and

$$
g_{1}(\xi)=-\frac{\pi}{2} .
$$

According to (3.30) the outging geometrical optics field can be written as

$$
u_{0}=\frac{1}{\sqrt{k}} \frac{g(\xi)}{\sqrt{\sigma-\xi}} e^{i k \sigma-i \frac{n_{1}}{2} g^{2}(\xi) \log (\sigma-\xi)-i \frac{\pi}{2}} .
$$

From the above results, we know that the incident and outgoing geometrical optics fields have the same amplitude term. If we compare the phase terms in (3.27) and (3.62), we find that the difference between two phase terms is an amount of $\frac{\pi}{2}$. It means that, when an incident beam is passing through the caustic region, it must undergoes a phase shift by an amount of $\frac{\pi}{2}$ and then becomes an outgoing beam. Similar result can also be found in the linear theory.

\section{References}

[1] E.L. Ince, Ordinary Differential Equations, New York, Dover Publications, 1956.

[2] M.J. Ablowitz and H. Segur, Solitons and the Inverse Scatering Transform, Philadelphia, SIAM, 1981.

[3] J.W. Miles, "On the Second Painlevé Transcendent", Proc. R. Soc. Lond. A361 (1978) 277-281. [4] C.M. Bender and S.A. Orszag, Advanced Mathematical Methods for Scientists and Engineers, New
York, McGraw-Hill, 1978. 